\title{
Bayesian inversion of Stokes profiles ${ }^{\star}$
}

\author{
A. Asensio Ramos ${ }^{1}$, M. J. Martínez González ${ }^{2}$, and J. A. Rubiño-Martín ${ }^{1}$ \\ 1 Instituto de Astrofísica de Canarias, 38205, La Laguna, Tenerife, Spain \\ e-mail: aasensio@iac.es \\ 2 LERMA, Observatoire de Paris-Meudon, 5 place Jules Janssen, 92195 Meudon, France
}

Received 18 June 2007 / Accepted 22 August 2007

\begin{abstract}
Context. Inversion techniques are the most powerful methods to obtain information about the thermodynamical and magnetic properties of solar and stellar atmospheres. In the recent years, we have witnessed the development of highly sophisticated inversion codes that are now widely applied to spectro-polarimetric observations. The majority of these inversion codes are based on the optimization of a complicated non-linear merit function. The experience gained has facilitated the recovery of the model that best fits a given observation. However, and except for the recently developed inversion codes based on database search algorithms together with the application of Principal Component Analysis, no reliable and statistically well-defined confidence intervals can be obtained for the parameters inferred from the inversions.

Aims. A correct estimation of the confidence intervals for all the parameters that describe the model is mandatory. Additionally, it is fundamental to apply efficient techniques to assess the ability of models to reproduce the observations and to determine to what extent the models have to be refined or can be simplified.

Methods. Bayesian techniques are applied to analyze the performance of the model to fit a given observed Stokes vector. The posterior distribution, that takes into account both the information about the priors and the likelihood, is efficiently sampled using a Markov chain Monte Carlo method. For simplicity, we focus on the Milne-Eddington approximate solution of the radiative transfer equation and we only take into account the generation of polarization through the Zeeman effect. However, the method is extremely general and other more complex forward models can be applied, even allowing for the presence of atomic polarization.

Results. We illustrate the method with different problems, from academic to more realistic examples. We show that the information provided by the posterior distribution is fundamental to understand and determine the amount of information available in the Stokes profiles in these particular cases.
\end{abstract}

Key words. magnetic fields - Sun: atmosphere - Sun: magnetic fields - line: profiles - polarization

\section{Introduction}

One of the most important breakthroughs in the interpretation of spectro-polarimetric observations has been the development and systematic application of inversion techniques (see e.g., Bellot Rubio 2006, and references therein). They have allowed us to extract as much information as possible from the observed Stokes profiles. A model that is assumed to be successful in describing the astrophysical plasma is defined by a set of parameters, usually associated with interesting physical quantities. It can happen that these physical parameters are not direct observables that can be obtained directly from the Stokes profiles. Then, inversion techniques adjust the parameters that characterize the selected model so that the emergent Stokes profiles reproduce, as well as possible, the observed profiles.

The initial steps in the development of inversion codes were limited by the computational time required. These inversion techniques often need the application of time-consuming nonlinear optimization methods. For this reason, the first generation of inversion codes either used very simple models to reproduce the observed Stokes profiles or introduced some additional physical ingredients in the inversion scheme so that the complications were greatly reduced (e.g., Auer et al. 1977; Keller et al. 1990). This is the reason why simple Milne-Eddington atmospheres (ME, Auer et al. 1977; Landi Degl'Innocenti \& Landolfi 2004) have been widely applied for the retrieval of an average

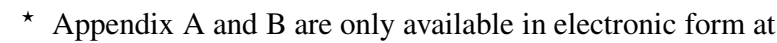
http://www . aanda. org. magnetic field vector in the line formation region. Although the assumptions on which ME atmospheres are based may not be exactly fulfilled in the solar atmosphere, they have been extensively used. The reason is their inherent simplicity and the fact that there is an analytic expression for the emergent Stokes profiles in terms of the physical parameters.

A great leap forward was the development of inversion codes based on the concept of response functions (Ruiz Cobo $\&$ del Toro Iniesta 1992). They have facilitated the inversion of Stokes profiles so that it is now possible to infer the vertical stratification of the thermodynamic and magnetic properties of the atmosphere if the information is present in the Stokes profiles. The presence of vertical variations along the line-of-sight of the physical properties are important in explaining the strong asymmetries observed in sunspots and faculae (Illing et al. 1975; Sanchez Almeida et al. 1989).

The development of such powerful and computationally efficient inversion codes has led to an extensive number of applications to a large variety of solar atmospheric structures (e.g., Westendorp Plaza et al. 1997; Sanchez Almeida 1997; Lites et al. 1998; Mathew et al. 2003; Bommier et al. 2007). Inversion codes optimise a given merit function with respect to a set of parameters. It is important, however, to be cautious regarding several fundamental points. First, the number of free parameters cannot be as large as desired. The reason is that the amount of information available in the observed Stokes profiles might not be enough to constrain the value of many of these parameters. Reasons for this include the presence of noise that masks 
the line profile dependence on certain parameters or the fact the Stokes parameters are insensitive to a parameter due to the intrinsic line formation process. Second, the parameters that we use to describe a given model might not be completely independent so that there exists (possibly nonlinear) combinations of these parameters that give rise to the same emergent Stokes profiles. Among these degeneracies, we can find the well-known ambiguity associated with the projection of the magnetic field vector on the plane of the sky, the degeneracy between the filling factor and the longitudinal component of the magnetic field strength (the magnetic flux density) in the weak-field regime and less-known degeneracies between thermodynamical and magnetic parameters for magnetic field structures organized on small scales (Martínez González et al. 2006). Third, since the optimization problem is usually solved with the aid of gradient descent methods like the Levenberg-Marquardt scheme, the solution given by the inversion code might not be that corresponding to the global minimum (if a global minimum is present).

Recently, several works have tackled this problem from different points of view. On the one hand, Asensio Ramos (2006) has introduced the use of model selection algorithms for the interpretation of spectro-polarimetric observations. Given a set of possible models that can be used to describe the observations, these algorithms help us select the most probable one using a quantitative approach. These algorithms, based on Occam's Razor, favor models that better fit the observations with a reduced set of parameters, while disfavoring too complicated models even if they match the observations or those that badly fit the observables. On the other hand, Asensio Ramos et al. (2007b) have applied algorithms based on geometric considerations to estimate the intrinsic amount of information present in the Stokes profiles. The intrinsic dimension of the manifold in which the observables lie can be associated with the number of independent free parameters that can be used when proposing a model to describe the observations. They have also shown that the amount of information present in an observed dataset increases monotonically with the number of spectral lines included. Also of interest is the work of Socas-Navarro (2004b) for estimating the level of detail of the stratification of atmospheric parameters one can obtain from selected spectral lines.

An important advance in the development of inversion codes was the application of database search algorithms in conjunction with Principal Component Analysis (PCA) to the inversion of Stokes profiles (Rees et al. 2000; López Ariste \& Casini 2002; Skumanich \& López Ariste 2002; Casini et al. 2005). This inversion technique only has been applied to simplified ME atmospheres and to microstructured magnetic atmospheres (MISMA; Socas-Navarro \& Sánchez Almeida 2002) but nothing (except for a computational problem) precludes using more complicated models. It is based on the direct comparison between the observed Stokes profiles and all the possible ones that can be built by varying the parameters that describe the model atmosphere. This comparison is not done with the profiles themselves, but with the coefficients of the projection of both the observed and theoretical profiles into a given basis. The key point of the PCA inversion is that this basis set is obtained from the synthetic Stokes profiles themselves. Consequently, it already encodes valuable information about the line formation mechanism. The fact that we compare the observed Stokes profile with the whole database allows us to reach the global minimum, instead of getting stuck in local minima. A by-product of using a database is that it is possible to define an error bar and give uncertainties on the inferred physical parameters.
This paper addresses the development of an inversion scheme that allows one to characterize the probability distribution of parameters of the model that better fit the observed Stokes profiles. To this end, we adopt a Bayesian approach to infer the most probable values of the parameters and to extract their confidence levels.

\section{Bayesian inversion of Stokes profiles}

Our aim is to develop an inversion code that can obtain all the physical information present in the observed Stokes profiles and that can give us detailed statistical information. This statistical information allows us to estimate a real error bar for each parameter and whether a parameter of a given model is constrained by the observables or not. This information turns out to be fundamental so that one can trust the value of the inferred parameters and properly analyze the observations.

\subsection{Forward modeling}

The Bayesian formalism is extremely general and can be applied to any model that explains a given set of observations. We are interested in the Stokes profiles emerging from a given atmosphere. Let $\boldsymbol{S}=(I, Q, U, V)^{\dagger}$ be the Stokes vector ( $\dagger$ indicating transpose). The vectorial radiative transfer equation describes the variation along a given ray of the Stokes vector $S$ depending on the absorption and emission properties of the medium:

$\frac{\mathrm{d} S}{\mathrm{~d} s}=\epsilon-\mathbf{K} S$

where $\epsilon=\left(\epsilon_{I}, \epsilon_{Q}, \epsilon_{U}, \epsilon_{V}\right)^{\dagger}$ is the emission vector and $\mathbf{K}$ is the propagation matrix:

$\mathbf{K}=\left(\begin{array}{cccc}\eta_{I} & \eta_{Q} & \eta_{U} & \eta_{V} \\ \eta_{Q} & \eta_{I} & \rho_{V} & -\rho_{U} \\ \eta_{U} & -\rho_{V} & \eta_{I} & \rho_{Q} \\ \eta_{V} & \rho_{U} & -\rho_{Q} & \eta_{I}\end{array}\right)$

In principle, once $\epsilon$ and $\mathbf{K}$ are known for all the points along the considered ray, it is possible to solve Eq. (1) and obtain the synthetic emergent Stokes parameters. However, for simplicity, we will focus on the Milne-Eddington approximation, although we plan to apply Bayesian inversion techniques to other more complex problems. Of interest is the case of inversion under local thermodynamic equilibrium (LTE; Ruiz Cobo \& del Toro Iniesta 1992) in which strong degeneracies may be present (see Martínez González et al. 2006) and the case of scattering polarization and the Hanle effect with many (and even unknown) degeneracies (House 1977; Casini \& Judge 1999; Trujillo Bueno 1999, 2001; Casini et al. 2005). In the ME approximation (see, e.g., Auer et al. 1977; Landi Degl'Innocenti \& Landolfi 2004), we assume that the ratio between the line absorption coefficient and the continuum absorption coefficient does not vary with depth in the atmosphere and that the line source function has a linear dependence on the optical depth along the line-of-sight. Furthermore, we assume that the magnetic field vector $\boldsymbol{B}$ and the bulk velocity are constant with depth.

Here we focus on the Zeeman effect as the mechanism that generates and modifies the polarization state of the atmosphere. In this case, the elements of the propagation matrix and of the emission vector can be easily calculated (e.g., Landi Degl'Innocenti \& Landolfi 2004). These elements depend on the strength of the magnetic field and on the specific orientation of 
the field vector with respect to the line-of-sight. After these assumptions, the well-known Milne-Eddington analytical solution of the radiative transfer equation can be applied. In the code, the effect of the magnetic field on the energy levels can be treated under the simple linear Zeeman regime, under the more general incomplete Paschen-Back regime or even hyperfine structure can be included.

\subsection{Posterior probability}

The interest in extracting all the information available in the observations has led to the systematic application of methods based on the Bayesian approach. A myriad of problems can be tackled within this framework that has strong theoretical roots. We present the fundamental ideas of the formalism, although more detailed information can be found in several monographs (see e.g., Neal 1993). Let us assume a model $M$ that is used to describe a given dataset $D$. In our case, the model $M$ is the Milne-Eddington approximation. It is parameterized in terms of the vector of physical quantities $\boldsymbol{\theta}$ that contains the usual ME parameters: Doppler width of the line in wavelength units $\left(\Delta \lambda_{\text {dopp }}\right)$, wavelength shift due to the macroscopic bulk velocity $\left(v_{\text {mac }}\right)$, gradient of the source function $(\beta)$, ratio between the line and continuum absorption coefficients $\left(\eta_{0}\right)$, line damping parameter (a) and magnetic field vector parameterized by its modulus, inclination and azimuth with respect to a given reference direction $\left(B, \theta_{B}\right.$ and $\phi_{B}$, respectively). It is customary to have some initial information about the physical parameters. For instance, an estimation of the range of variation of the physical parameters might be available, although sometimes it can be a very rough one (for instance, a limitation to positive or negative values). This information is incorporated into a prior distribution $p(\boldsymbol{\theta})$. When the information contained in the data $D$ is incorporated in the problem, our state of knowledge of the parameters changes according to the Bayes theorem:

$p(\boldsymbol{\theta} \mid D) \propto p(\boldsymbol{\theta}) p(D \mid \boldsymbol{\theta})$.

The posterior distribution $p(\boldsymbol{\theta} \mid D)$ represents our state of knowledge of the parameters once the information of the dataset has been taken into account. The term $p(D \mid \boldsymbol{\theta})$ is the so-called likelihood function and gives information about how well a particular set of parameters predicts the observed data. The Bayes theorem states that whether a model $M$ becomes plausible after the data $D$ has been taken into account depends on how plausible the model was before taking into account the data and how well the model predicts the data. The simplicity of the Bayes theorem hides all its potential and this kind of reasoning has led to a variety of applications: it has been widely used in cosmological analyses (e.g., Lewis \& Bridle 2002; Rubiño-Martin et al. 2003; Rebolo et al. 2004), gravitational wave analyses (e.g., Cornish \& Crowder 2005), gravitational lensing (e.g., Brewer \& Lewis 2006), oscillation of solar-like stars (e.g., Brewer et al. 2007), analysis of solar extreme-ultraviolet spectra (e.g., Kashyap \& Drake 1998) and many more. A powerful inversion code can be built based on the Bayes theorem. Once the posterior distribution $p(\theta \mid D)$ is known, the position of the maximum value gives the most probable combination of parameters that fit the data. Not only this, but we can also analyze the confidence of the parameters. Consequently, degeneracies, ambiguities and the rest of problems that arise in typical inversion codes (except for those based on PCA) can be investigated in detail.

Let us analyze in detail the terms appearing on the right hand side of in Eq. (3). As mentioned above, the prior distribution contains all the information that we know about the parameters without taking into account the observed data. In the most simple case, we can assume that all the parameters are statistically independent, so that the prior distribution can be written as:

$$
p(\boldsymbol{\theta})=\prod_{i=1}^{N_{\mathrm{par}}} p\left(\theta_{i}\right)
$$

where the $\left\{\theta_{i}\right\}$ are the parameters included in the model and $N_{\mathrm{par}}$ is the number of such parameters. Unless physical information is available, we typically only know the range of variation of the parameters, so that we can write:

$p\left(\theta_{i}\right)=H\left(\theta_{i}, \theta_{i}^{\min }, \theta_{i}^{\max }\right)$

where $H(x, a, b)$ is the top-hat function:

$$
H(x, a, b)=\left\{\begin{array}{cc}
\frac{1}{b-a} & a<x<b \\
0 & \text { otherwise }
\end{array}\right.
$$

As an example, consider the prior of a uniform magnetic field vector $\boldsymbol{B}$. In order to guarantee such uniform magnetic field vector, we have to sample uniformly the volume element $\mathrm{d} V=r^{2} \mathrm{~d} r \mathrm{~d}\left(\cos \theta_{B}\right) \mathrm{d} \phi_{B}$. We can assume that the magnetic field strength cannot be larger than $B_{\max }$, so that its prior is a top-hat function that is non-zero in the interval $\left[0, B_{\max }^{3}\right]$. When focusing on the solar atmosphere, a reasonable choice is $B_{\max } \approx 4000 \mathrm{G}$ so that all the physically relevant cases can be covered (quiet Sun, sunspots, faculae, etc.). The inclination and the azimuth have to be limited to the ranges $[0, \pi]$ and $[0,2 \pi]$, respectively. If we neglect any correlation between the magnetic field strength, inclination and azimuth, the final prior on the magnetic field vector is given by:

$$
p(\boldsymbol{B})=H\left(B^{3}, 0, B_{\max }^{3}\right) H\left(\cos \theta_{B},-1,1\right) H\left(\phi_{B}, 0,2 \pi\right) .
$$

Interestingly, it is possible to include correlations between the parameters. As an example, let us assume that the stronger the magnetic field, the more vertical it is. Additionally, weaker fields can be found with all kinds of inclinations. A simple prior distribution that fulfills the previous assumptions is:

$$
\begin{aligned}
p(\boldsymbol{B}) & =\frac{1}{C}\left\{1+\exp \left[-\left(B-B_{\max }\right)^{2} / \sigma_{\mathrm{B}}^{2}\right] \exp \left[-\theta_{B}^{2} / \sigma_{\theta}^{2}\right]\right\} \\
& \times H\left(\phi_{B}, 0,2 \pi\right)
\end{aligned}
$$

where $\sigma_{\mathrm{B}}^{2}$ and $\sigma_{\theta}^{2}$ control the shape of the prior and $C$ is a normalization constant.

The second term, $p(D \mid \boldsymbol{\theta})$, termed the likelihood, measures the probability that a model determined by a set of parameters $\theta$ fits a given observation $D$. To simplify the notation, it is advantageous to particularize to the case of the inversion of Stokes profiles. In spite of this particularization, the method still remains very general. The data $D$ that we are facing consists of a set of four vectorial quantities, i.e., the wavelength dependence of the four Stokes parameters. The number of wavelength points in each Stokes profile is indicated by $N_{\lambda}$. The value of the Stokes parameter $i=0,1,2,3$ (that we associate with the more usual notation $I, Q, U$ and $V$ ) at a wavelength $\lambda_{j}$ is represented by the quantity $S_{i}^{\text {obs }}\left(\lambda_{j}\right)$. When these Stokes parameters are observed with a spectro-polarimeter attached to a telescope, they contain a certain level of noise. If these observational errors are independent and have a Gaussian distribution, their distributions can be described by their standard deviations $\sigma_{i}\left(\lambda_{j}\right)$, i.e., the noise level for each Stokes parameter at wavelength $\lambda_{j}$. Strictly speaking, the noise in the observed Stokes profiles should be Poissonian 
because it comes mainly from photon noise. However, for consistency with other works, we choose the noise to be normally distributed, which will be a good approximation if the number of photons is high enough. Typically, we will deal with wavelengthindependent noise, so that only the four quantities $\sigma_{i}$ are needed. Let $S_{i}^{\text {syn }}\left(\lambda_{j}\right)$ be the Stokes parameters that emerge when the forward problem is solved in a given model $M$ parameterized by the vector of parameters $\boldsymbol{\theta}$. Taking into account the previous definitions and assumptions, the likelihood function is defined as (e.g., MacKay 2003):

$p(D \mid \boldsymbol{\theta}) \propto \mathrm{e}^{-\frac{1}{2} \chi^{2}}$,

where we have introduced the usual merit function $\chi^{2}$ :

$\chi^{2}=\frac{1}{4 N_{\lambda}} \sum_{i=1}^{4} \sum_{j=1}^{N_{\lambda}}\left(\frac{S_{i}^{\mathrm{obs}}\left(\lambda_{j}\right)-S_{i}^{\mathrm{syn}}\left(\lambda_{j}\right)}{\sigma_{i}}\right)^{2}$.

Although we have focused on wavelength-independent noise, the formalism allows us to accommodate wavelength-dependent noise by using $\sigma_{i}\left(\lambda_{j}\right)$ instead of $\sigma_{i}$ in the likelihood. Therefore, if the information is available, it is possible to include other sources of uncertainty like reduction residuals, cross-talk, fringes, etc. The $\chi^{2}$ function that is typically used for the inversion of Stokes profiles presents the weights $\left\{w_{i}, i=1 \ldots 4\right\}$ for each Stokes parameter. This differential weighting scheme is not applied here, but the method can accommodate it straightforwardly. The only influence of this weighting is to change the width of the maximum likelihood regions (reducing or expanding the confidence regions around the maximum). However, the location of the maximum is not changed.

\section{Markov chain Monte Carlo}

In the Bayesian framework, the most plausible model is the one that maximizes the posterior distribution. Our objective is then to sample the posterior distribution and to find the combination of parameters that produce this maximum value. This will represent the most plausible model that matches the observed Stokes profiles. For a small number of parameters $N_{\text {par }}$, this brute force approach might be achievable. For instance, assuming that ten values per parameter are desired, something like $10^{N_{\text {par }}}$ evaluations of the posterior distribution are needed. This implies that the forward model has to be evaluated a huge number of times. When $N_{\text {par }}<5$, such a direct approach can be applicable if the computing time per evaluation of the forward model is not very large. However, this brute force approach quickly becomes impractical because the number of function evaluations increases exponentially with the number of free parameters. In order to overcome this difficulty, we have applied a Markov chain Monte Carlo technique. Since this is the first time that such a method is applied to the inversion of Stokes profiles, we present in detail some important technical issues in Appendix A, although they are widely known in other research fields. Briefly, our implementation of the Markov chain Monte Carlo scheme is based on the Metropolis algorithm (Metropolis et al. 1953; Neal 1993). The proposal density distribution is chosen to be a multi-variate Gaussian with a diagonal covariance matrix. Our code uses the convergence criterium of Dunkley et al. (2005) although other criteria are discussed in the Appendix.

\section{Illustrative examples}

In order to show the capabilities of the newly developed code, several examples are shown. Some of them deal with synthetic data where we can investigate the behavior of the method under controlled conditions. After these synthetic tests, we apply the code to a realistic case obtained from spectro-polarimetric observations.

\subsection{Simple academic example}

The first example serves as an illustration of how the MCMC method is able to capture the presence of degeneracies. To this end, a very simplified example is presented, where we make use of a Zeeman triplet line, namely the Fe I line at $630.2 \mathrm{~nm}$. The emergent Stokes profiles are calculated in a Milne-Eddington atmosphere. The value of the parameters are: $\Delta v_{\text {dopp }}=2.4 \mathrm{~km} \mathrm{~s}^{-1}$, $v_{\text {mac }}=0 \mathrm{~km} \mathrm{~s}^{-1}, \beta=9, \eta_{0}=9.8, a=0.3, B=100 \mathrm{G}, \theta_{B}=45^{\circ}$ and $\phi_{B}=0^{\circ}$. The main characteristic of such synthetic profiles is that the magnetic field strength is so weak $(B=100 \mathrm{G})$ that the Zeeman splitting is negligible compared to the Doppler width of the line. As a consequence, the emergent Stokes profiles can be described in the weak field regime of the Zeeman effect, in which the Stokes $V$ profile is proportional to the wavelength derivative of the intensity profile (e.g., Landi Degl'Innocenti 1992). It is widely known that only the line-of-sight component of the magnetic field vector (i.e., the product $B \cos \theta$, with $\theta$ the angle between the line-of-sight and the magnetic field vector) can be obtained from the amplitude of the Stokes $V$ profile. On the contrary, when linear polarization is also present, the precise magnetic field vector can also be obtained. Since linear polarization appears as a second order contribution to the emergent signal, they are difficult to observe and a reduced noise level is fundamental. In this section we present an analysis with the aid of the MCMC code of how the information retrieved from the previously described synthetic Stokes profiles degrades with the presence of noise. The noise is described by a Gaussian distribution parameterized by the value of $\sigma$, which here is given in units of the continuum intensity, $I_{\mathrm{c}}$.

We run the MCMC code on the synthetic Stokes profiles taking into account the full Stokes vector for the calculation of the likelihood function given by Eq. (9). All the thermodynamical parameters and the azimuth are assumed to be known and we only allow the magnetic field strength and the inclination of the field to vary. We test that the obtained Markov chain is converged for the two parameters as indicated in Appendix A. Although it depends on the complexity of the problem, our Markov chains require lengths of the order of 50000 accepted samples to fulfill the convergence criterion, with a total computational time of the order of 20 seconds on a standard computer. Finally, taking into account that the obtained Markov chain is sampling from the posterior probability distribution, the posterior itself can be obtained simply by "making histograms". Two different cases with different amounts of added noise have been considered. A case in which the added noise level is $\sigma=10^{-5}$, whose results are shown in Fig. 1, and a case with a much larger noise of $\sigma=10^{-3}$, whose results are shown in Fig. 2. The two-dimensional histograms shown in the right panels of both figures present a graphical representation of the posterior distribution of the magnetic field strength and inclination, $p\left(B, \theta_{B}\right)$. We show two contours indicating confidence levels of $68 \%$ and $95 \%$, respectively. The case $\sigma=10^{-5}$ shows a clearly peaked posterior distribution, indicating that a very good estimation of the magnetic field strength and inclination is possible. On the contrary, the case $\sigma=10^{-3}$ presents a clear degeneration between both parameters, manifested by the typical "banana-shaped" posterior distribution. The main reason for this extended posterior distribution is that the 

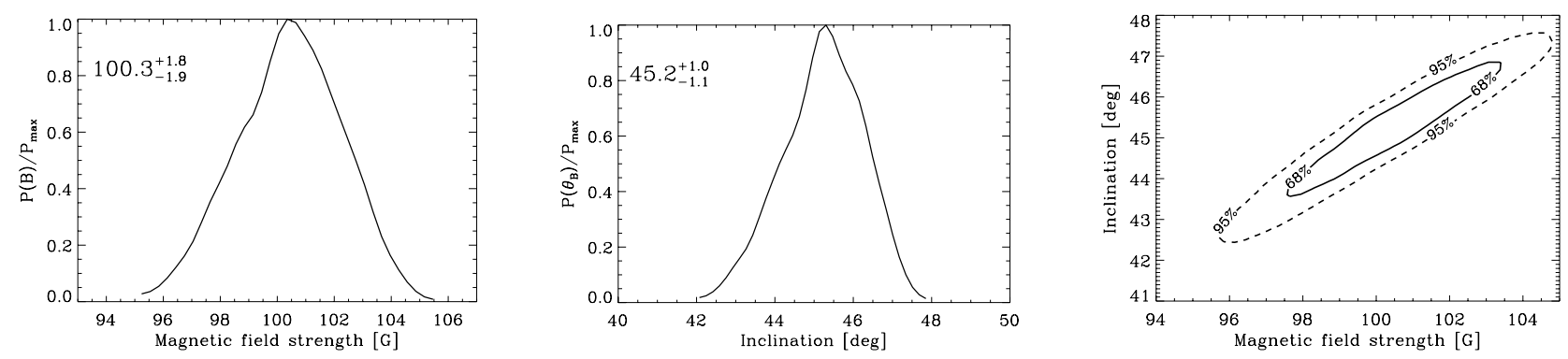

Fig. 1. Posterior probability distribution for the simple academic example with a noise $\sigma=10^{-5}$ in units of the continuum intensity, $I_{\mathrm{c}}$. The full Stokes vector $(I, Q, U, V)$ is taken into account. The left panel shows the marginalized distributions for the magnetic field strength while the central panel shows the distribution for the inclination. The right panel shows the tow-dimensional posterior distribution. The contours indicate the confidence levels at $68 \%$ (solid line) and 95\% (dashed line).
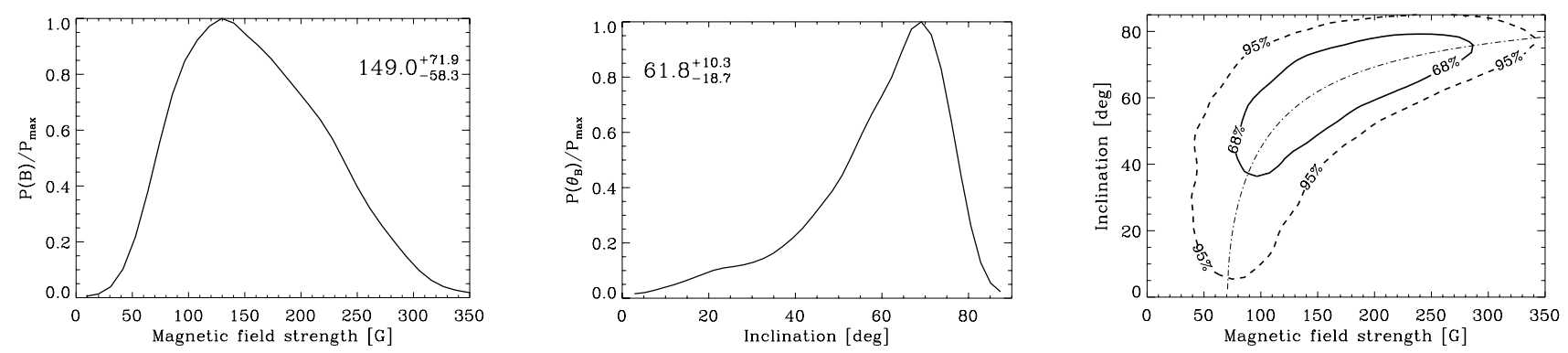

Fig. 2. Posterior probability distribution for the simple academic example with a noise $\sigma=10^{-3}$ in units of the continuum intensity. The posterior clearly shows a degeneracy between the magnetic field strength and the inclination. The dashed thin line in the right panel indicates the points where $B \cos \theta_{B}=B^{\prime} \cos \theta_{B}^{\prime}$, where the primed quantities are those belonging to the synthetic profile, namely, $B^{\prime}=100 \mathrm{G}$ and $\theta_{B}^{\prime}=45^{\circ}$.

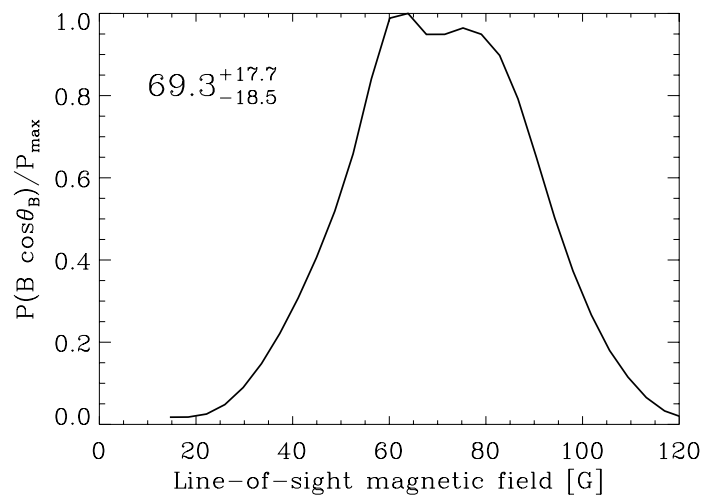

Fig. 3. Posterior probability distribution of the line of sight component of the magnetic field, $B \cos \theta_{B}$ for the simple academic example with a noise $\sigma=10^{-3}$ in units of the continuum intensity. The posterior clearly shows a peak compatible with the original value.

Stokes $Q$ and $U$ signals are masked below the noise level. For such a high noise level, only the information encoded in the Stokes $V$ signal is available for retrieving the magnetic field strength and inclination. Since the field is only $100 \mathrm{G}$, the line is in the weak-field regime so that only the product $B \cos \theta_{B}$ can be estimated from Stokes $V$. In order to make sure that this is indeed the case, we have overplotted the curve $B \cos \theta_{B}=B^{\prime} \cos \theta_{B}^{\prime}$ with $B^{\prime}=100 \mathrm{G}$ and $\theta_{B}^{\prime}=45^{\circ}$, which closely follows the shape of the posterior. Figure 3 shows the marginalized distribution of the line-of-sight component of the magnetic field, $B \cos \theta_{B}$, showing that it can be recovered with accuracy. Marginalized posteriors ${ }^{1}$ $P(B)$ and $P\left(\theta_{B}\right)$ are also shown in the left and central panels of Figs. 1 and 2. Sharp distributions are found for the case with $\sigma=10^{-5}$ noise, while distributions with enhanced tails are found

1 They are obtained by integrating the two-dimensional histogram with respect to one of the variables. for the case $\sigma=10^{-3}$. Curiously, according to the marginalized distributions, a reasonably estimation of the field strength is possible even for the highly noisy profiles, although there is a non-negligible tail for larger field strengths. The posterior $P\left(\theta_{B}\right)$ gives reduced information about the inclination, clearly showing the $B \cos \theta_{B}$ degeneracy. For comparison, we have applied an inversion code based on the Levenberg-Marquardt algorithm to estimate the parameters and their confidence intervals for the case with $\sigma=10^{-3}$. The minimum of the $\chi^{2}$ function is correctly obtained for $B=98.5 \mathrm{G}$ and $\theta_{B}=44.1^{\circ}$. However, the symmetric confidence intervals that we obtain using the diagonal elements of the covariance matrix (e.g., Press et al. 1986) produce an estimation of $98.5 \pm 180.9 \mathrm{G}$ for the magnetic field strength and $44.1 \pm 100.5$ degrees for the magnetic field inclination. According to the estimated error, the field inclination is not constrained by the observations. These results provide a poor estimation of the confidence intervals compared to the marginalized posterior distributions shown in Fig. 2.

The utility of this test is two-fold: on the one hand, we have demonstrated, with a simplified problem, the correct operation of the MCMC inversion code; on the other hand, we point out the obvious importance of having accurate Stokes profiles in order to recover information about the magnetic field vector.

\subsection{The problem of the quiet Sun}

After the simple instructive example, we now focus on a more realistic problem that presents deep implications for the recovery of information about the magnetism of the quiet solar photosphere. The quiet Sun are those regions away from the most evident manifestations of magnetic activity. In the photosphere, it corresponds mainly to the network (magnetic flux concentrations in the supergranular boundaries) and the internetwork (filling up the interior of supergranular cells). At the present 
spatial resolution of ground-based spectropolarimetric observations $\left(0.5-1^{\prime \prime}\right)$, the magnetic structures on the quiet Sun are thought to be not spatially resolved (e.g., Stenflo 1994; Lin 1995; Domínguez Cerdeña et al. 2003; Khomenko et al. 2003; Martínez González et al. 2006). This has been demonstrated by Asensio Ramos et al. (2007a), presenting the first map of flux cancellation in the quiet Sun. The magnetism of the network is widely established as predominantly vertical $\mathrm{kG}$ structures filling approximately $10-20 \%$ of the resolution element. However, the problem is more complicated in the internetwork, where the polarization signals that we can measure by means of the Zeeman effect are unresolved, occupying only the $1-2 \%$ of the resolution element. Typically the Stokes $V$ profiles have an amplitude of $10^{-3}$ in units of the continuum intensity, $I_{\mathrm{c}}$. The noise that we can achieve in the observational data $\left(\sim 10^{-4} I_{\mathrm{c}}\right)$ is only one order of magnitude smaller than the polarimetric signals in the internetwork. As it has been shown in the previous section, it is important to have a reduced noise level in order to obtain information about the magnetic field from the observed Stokes profiles. In the internetwork, when the widely observed pair of Fe 1 lines at $630 \mathrm{~nm}$ are used, no linear polarization signal above the noise level is found with the current instrumentation. However, even if there is a lack of signal in Stokes $Q$ and $U$, we could retrieve magnetic field strengths when the line is out of the weak field regime. In this particular pair of lines and for the typical photospheric physical conditions, the line can be considered in the weak field regime for fields below $\sim 600 \mathrm{G}$. This would mean that the $\mathrm{kG}$ magnetic field strengths retrieved from this pair of lines would be reliable (e.g., Grossmann-Doerth et al. 1996; Sigwarth et al. 1999; Domínguez Cerdeña et al. 2003; Sánchez Almeida et al. 2003).

However, Martínez González et al. (2006) have shown that these results should be considered with care. They show the most simple case in which the thermodynamics compensate for the effect of the magnetic field. These authors used the $\mathrm{SIR}^{2}$ code (Ruiz Cobo \& del Toro Iniesta 1992) to synthesize the emergent Stokes profiles using the typical physical conditions of the internetwork. The inversion of such a profile with random initializations showed that the resulting atmospheres depended on the initialization itself if a noise level of $5 \times 10^{-5} I_{\mathrm{c}}$ is assumed. In each case the change in the magnetic field was compensated for by a small change in the magnetic temperature gradient (smaller than $300 \mathrm{~K}$ ) and a slight increase of the microturbulent velocity (below $1.5 \mathrm{~km} \mathrm{~s}^{-1}$ ). The change in the temperature gradient produces a modification in the Stokes $V$ ratio of the two spectral lines while the increase in the microturbulent velocity leads to a broadening of the line profile. This procedure clearly demonstrated the degeneracy of the inversion problem in this particular case. Unfortunately, the Levenberg-Marquardt algorithm used in the SIR code for the inversion of Stokes profiles does not produce a reliable and well-defined estimation of the errors in the parameters that describe the atmosphere. This is the reason why Martínez González et al. (2006) showed the degeneracy of the inversion problem by using repeated inversions with random initializations. In this paper, we follow the study performed by Martínez González et al. (2006) and we extend it to the cases in which we increase the filling factor (we improve the signal to noise ratio) or we add a particular inclination to the magnetic field vector (we generate a linear polarization signal). However, our solutions are based on robust statistical techniques.

The interpretation of the weak field regime when the magnetic feature is resolved is straightforward. In the weak field

\footnotetext{
${ }^{2}$ Stokes Inversion based on Response functions.
}

approximation, the radiative transfer equation has an analytical solution (see Chapter 9 of Landi Degl'Innocenti \& Landolfi 2004, for the conditions under which this approximation is valid). The Stokes $V$ profiles can be written as:

$$
V(\lambda)=-4.6686 \times 10^{-13} \bar{g} \lambda_{0}^{2} B \cos \theta \frac{\partial I(\lambda)}{\partial \lambda},
$$

where $\bar{g}$ is the effective Lande factor of the line, $\lambda_{0}$ is the central wavelength of the spectral line given in $\AA$ and $B$ is the magnetic field strength given in $\mathrm{G}$. The simultaneous observation of the Stokes $I$ and $V$ profiles allows us to compute the product $B \cos \theta$. The situation in the quiet Sun is not so straightforward since the magnetic structures occupy a very small portion of the resolution element. Then, the modeling of these areas requires at least two components: a magnetic component that gives rise to the polarization signals and a non-magnetic one that accounts for the rest of the pixel that is field-free ${ }^{3}$. Two complicated problems arise due to this particularity. First, the right-hand side of Eq. (11) has to be multiplied by the filling factor and the value that we will recover would be the longitudinal magnetic flux density $\alpha B \cos \theta$. Second, the intensity profile that applies in Eq. (11) comes from the magnetic component. Then, the product $\alpha B \cos \theta$ cannot be computed from the ratio of the Stokes $V$ profile and the wavelength derivative of Stokes $I$, since the observed Stokes $I$ comes mainly from the non-magnetic component. If one still wants to use the previous approach, the only way to recover the product $\alpha B \cos \theta$ would be by computing a calibration curve. This means that we have to assume a model atmosphere and compute the Stokes $V$ profile for different values of the longitudinal magnetic flux density. As a result, the inferred magnetic field is model dependent. In other words, the Stokes $V$ profiles depend on the magnetic and thermodynamic properties so, if one wishes to infer the magnetic properties of the plasma, it is fundamental to fix the thermodynamical properties first. The only technique available to overcome this difficulty is to apply inversion techniques. However, one has to have in mind that the information encoded in the Stokes I profile (that has $\sim 99 \%$ contribution from the nonmagnetic component) and in the Stokes $V$ profile is not enough to constrain the problem and to recover in a reliable way all the atmospheric parameters in a two component model.

\subsubsection{Recovering the magnetic field strength in the internetwork}

Here we use synthetic profiles that can be representative of the quiet Sun to see how well we can recover the magnetic field separately from the rest of the parameters. We synthesize the Fe I lines at 630.1 and $630.2 \mathrm{~nm}$ using a two component model. Both atmospheres have the same values of the ME parameters except for the magnetic field strength and the filling factor. The value of the parameters are: $\Delta v_{\text {dopp }}=0.05 \AA, v_{\text {mac }}=0 \mathrm{~km} \mathrm{~s}^{-1}$, $\eta_{0}^{630.1}=5.0, \eta_{0}^{630.2}=4.5, a=0.45 \AA$, and $\beta=8$. The magnetic flux density is fixed to $10 \mathrm{Mx} / \mathrm{cm}^{2}$, representative of the typical value in the internetwork. Since the magnetic field strength is set to $1000 \mathrm{G}$ and it has been assumed to be vertical, the filling factor of the magnetic component is set to $1 \%$. A certain amount of noise, characterized by a normal distribution with a standard deviation of $10^{-4} I_{\mathrm{c}}$, is added to the profiles.

3 The term field-free might be confusing since this component can indeed present a magnetic field that, due to its special structure, presents a zero Zeeman signal (e.g., microturbulent distribution, isotropic distribution, etc.). 

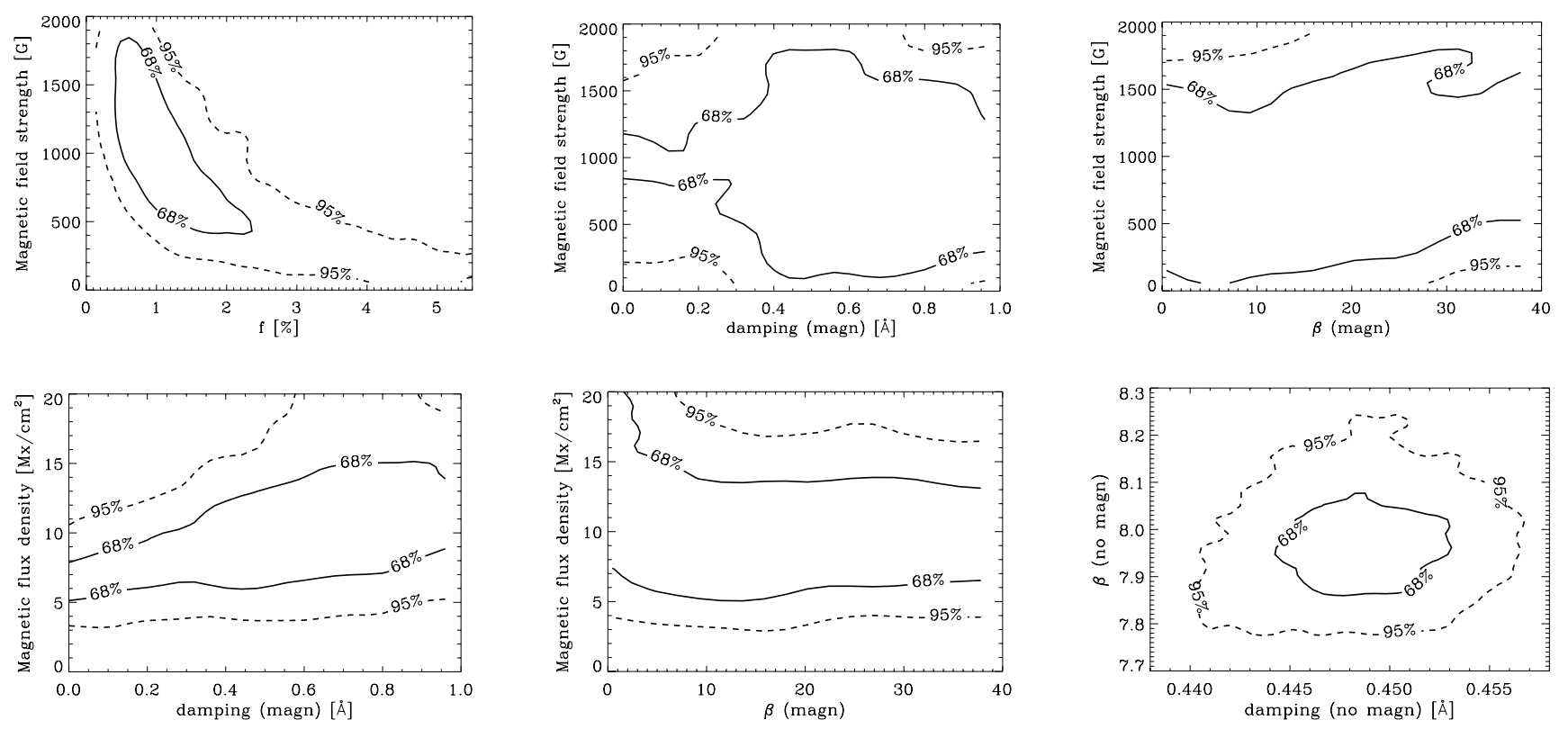

Fig. 4. Two-dimensional posterior distributions for several combinations of the parameters for the internetwork synthetic example with a longitudinal magnetic flux density of $10 \mathrm{Mx} / \mathrm{cm}^{2}$. The contours indicate the regions where $68 \%$ and $95 \%$ confidence levels are placed. Large degeneracies are present in almost all the parameters, except for the gradient of the source function and the damping in the non-magnetic component.

In a Milne-Eddington atmosphere, none of the parameters is strictly equivalent to the temperature or the microturbulent velocity that are present in the LTE approximation used by SIR. Accordingly, we select the gradient of the source function, $\beta$, and the damping coefficient, $a$, in both components together with the magnetic field strength and the filling factor as the free parameters in our test. Figure 4 summarizes the results of the Bayesian inversion. All the upper panels show the very high degree of degeneracy between the magnetic field strength and the rest of parameters. The upper left panel indicates that magnetic fields with all values below $2000 \mathrm{G}$ can reproduce the profile with an accuracy better than two times the noise level. Furthermore, magnetic field strengths between 400 and $1800 \mathrm{G}$ fit the profile with a confidence level smaller than the noise level (see an example of two possible fits with different field strengths in Appendix B). An interesting behavior is shown in the central and upper right panels. The posterior distribution presents almost no variation along these directions (damping parameter and gradient of the source function) and they are only limited by the ranges that we have assumed for them. Therefore, this means that the data has provided no new information for constraining these parameters (flat likelihood) and we are only recovering information about the priors. This is a typical example in which, due to the lack of information, the result depends critically on the prior information and one should be very cautious with the conclusions inferred from the calculations. Finally, the lower left and central panels show the marginalized posterior distribution for the longitudinal magnetic flux density and the damping $a$ and the gradient of the source function $\beta$, respectively. Again we see that the line profiles carry reduced information about these parameters. Even more striking is the fact that the longitudinal magnetic flux density is recovered with $\sim 50 \%$ error at a $68 \%$ confidence level. On the contrary, the parameters of the non-magnetic component are recovered with precision, as stated in the lower right panel of Fig. 4, with differences with respect to the input values that are well below $2.5 \%$ in both cases.

The previous analysis demonstrates that it is difficult to obtain reliable information from Stokes profiles representative of internetwork regions. However, what happens in strongly magnetized areas like the network, where the magnetic fluxes are 10-20 times higher than in the internetwork? To investigate this issue, we use the same ME parameters but we assume an enhanced magnetic flux density of $200 \mathrm{Mx} / \mathrm{cm}^{2}$, where we have increased the filling factor of the magnetic component to $20 \%$. The marginalized posterior distributions are shown in Fig. 5. In this case, both the magnetic field strength and the longitudinal magnetic flux density are well recovered, together with the damping, $a$. However, as a consequence of the reduced filling factor of the non-magnetic component with respect to the internetwork case, the gradient of the source function of the non-magnetic component presents a more extended posterior distribution. This behavior is easy to understand because there is less information about the non-magnetic component encoded in the Stokes profiles produced by the large filling factor of the magnetic component.

\subsubsection{Inclined fields}

It is of interest to investigate the shape of the marginalized posterior distributions when the magnetic field vector is inclined with respect to the line of sight. In this case, the information provided by the linear polarization profiles can lead to better constraints. We use the same synthetic profile with a magnetic flux density of $10 \mathrm{Mx} / \mathrm{cm}^{2}$ and we assume inclinations of $20^{\circ}, 45^{\circ}$ and $70^{\circ}$. In the first case, the Stokes $Q$ signal is below the noise level and it is not surprising that the results are comparable to the ones in which the magnetic field vector was assumed to be vertical. The case of an inclination of $70^{\circ}$ shows the same behavior since, in this case, the Stokes $V$ signal is below the noise level. Figure 6 shows the results of the inversion for the intermediate case of $\theta=45^{\circ}$. In this case, strong signatures of degeneracy are detected. The upper left panel shows that the magnetic field strength is concentrated in high values. However, this does not mean that the value of the field strength is better recovered. First, we can see the large degeneracy with the other parameters. Second, Fig. 7 shows that the marginalized posterior distribution for the magnetic field strength strongly resembles that 

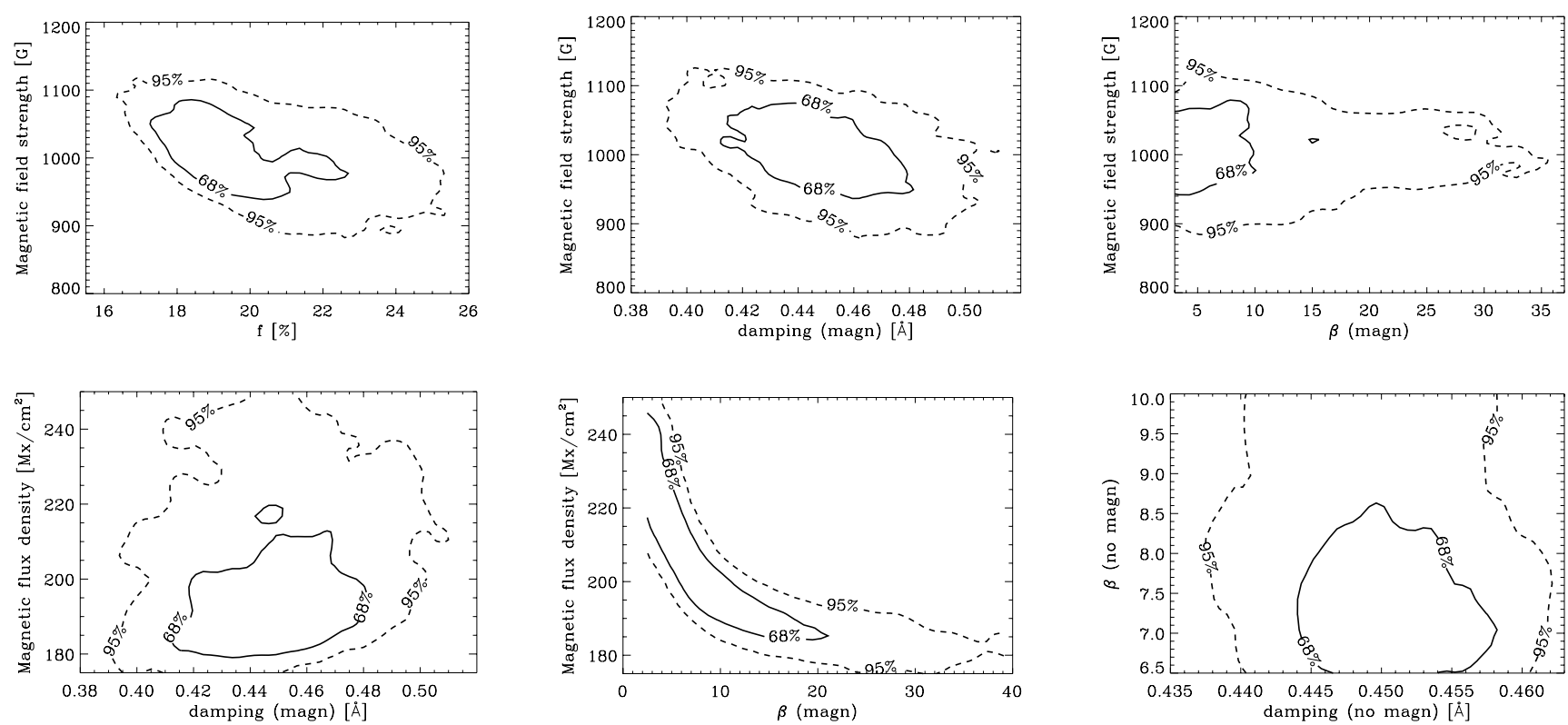

Fig. 5. Two-dimensional posterior distributions for several combinations of the parameters for the network synthetic example with a longitudinal magnetic flux density of $200 \mathrm{Mx} / \mathrm{cm}^{2}$. The contours indicate the regions where $68 \%$ and $95 \%$ confidence levels are placed. The magnetic field strength can be recovered better than in the internetwork case shown in Fig. 4. However, increased degeneracies are also seen in the parameters of the non-magnetic component due to the reduced surface covered by this component.
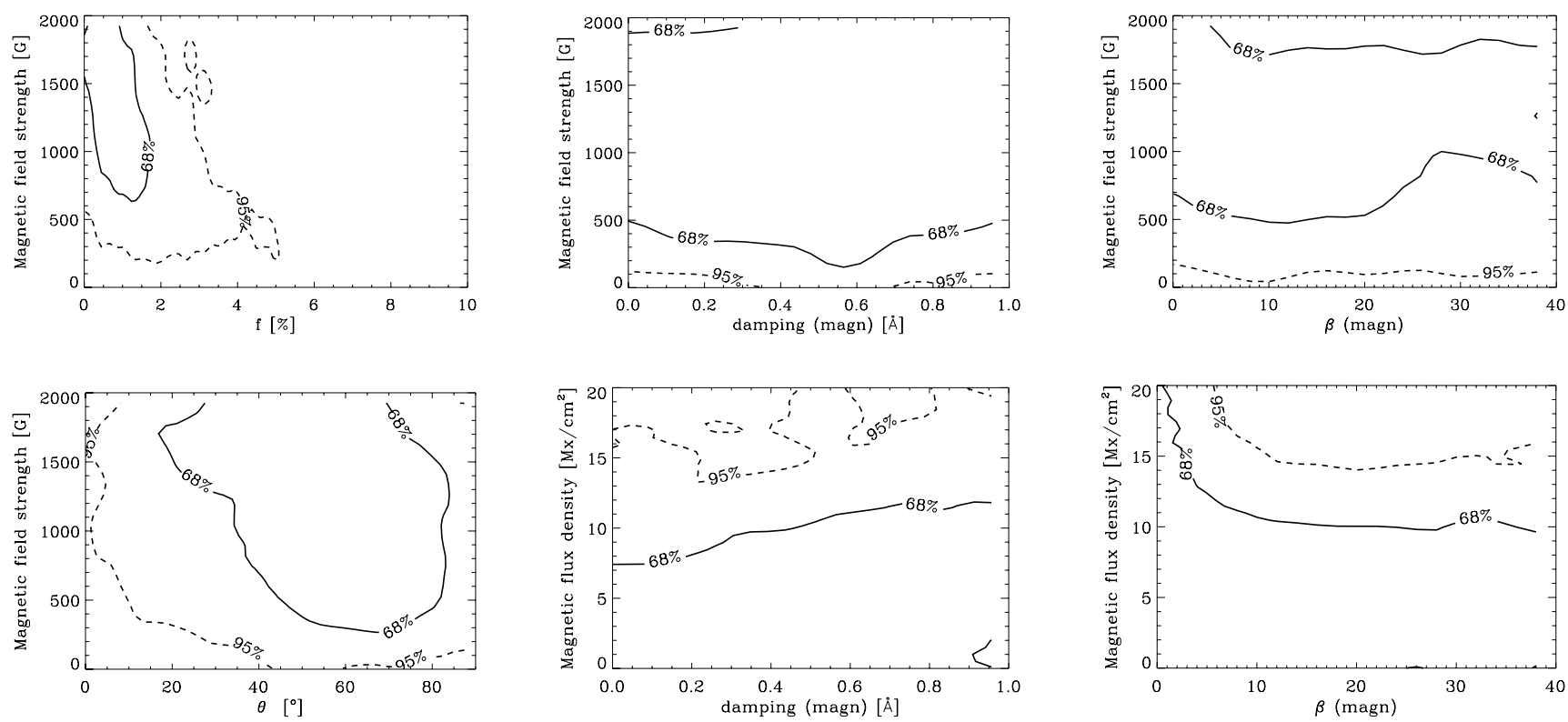

Fig. 6. Two-dimensional posterior distributions for several combinations of the parameters for the internetwork synthetic example when the magnetic field vector has an inclination of $45^{\circ}$. The contours indicate the regions where $68 \%$ and $95 \%$ confidence levels are placed. In spite of the presence of more information encoded in the linear polarization Stokes profiles, the marginalized posterior distributions show shapes very similar to those found in Fig. 4.

of the prior. It seems that even the inclination angle cannot be constrained with the available information. One of the reasons is that, although the Stokes $Q$ and $U$ signal might become larger than the noise, the Stokes $V$ signal decreases and gets closer to the noise. Therefore, some information available in Stokes $V$ is hidden by the presence of noise.

\subsection{Realistic examples}

In order to demonstrate the capabilities of the MCMC code, we show an application to realistic Stokes profiles. They correspond to a position on an umbra of a sunspot observed during
August 17, 2004 (Sainz Dalda \& López Ariste 2007). The observation was carried out with the THÉMIS telescope at the Observatorio del Teide (Spain). The telescope was operated in the MTR mode, so that the polarization analysis was performed for each wavelength at each pixel. Although the observation consisted of a scan over a sunspot, for the purpose of demonstrating the capabilities of the MCMC code, we only focus here on the information obtained in one pixel of the whole scan. The observed spectral region contains the previously mentioned $630 \mathrm{~nm}$ pair of Fe I lines. Figure 9 presents the observed Stokes profiles. The noise level estimated from the continuum where no polarization signal is detected is of the order of $1.6 \times 10^{-3}$ in units of 


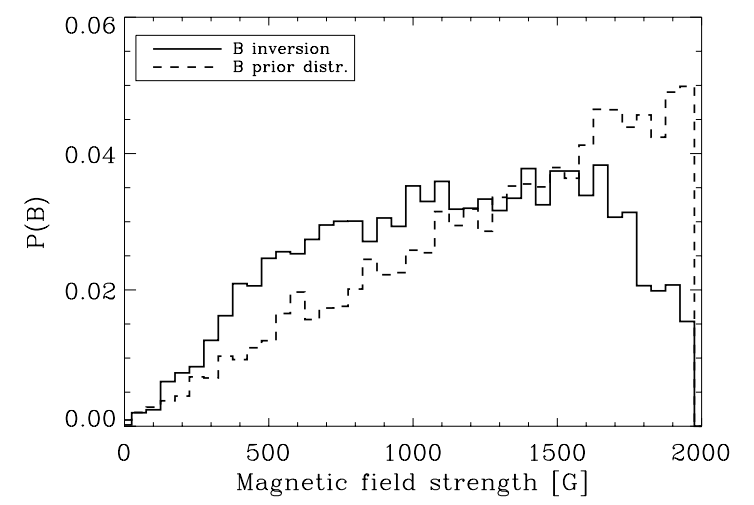

Fig. 7. Marginalized distribution of the inferred magnetic field strength (solid line) together with the prior one (dashed line). Both distributions are very similar, making us consider that the information contained in the emergent line profiles is very small.

the continuum intensity. This spectral region consists of two Fe I lines at 630.1 and $630.2 \mathrm{~nm}$, together with two telluric contributions. The wavelength calibration has been carried out with the aid of the two telluric lines. The $630.2 \mathrm{~nm}$ line presents a higher magnetic sensitivity and this translates into an enhanced Zeeman splitting that also can be detected in the Stokes I profile.

The MCMC code has been applied to both spectral lines separately. We leave all the Milne-Eddington parameters free but we only focus on the results concerning the magnetic field vector. Stray-light contamination from the surrounding quiet Sun is also taken into account. The results indicate a filling factor of the umbral component in the range $91-94 \%$, with a confidence interval of the order of $\pm 3 \%$. Figure 8 shows the results obtained from the inversion of the $630.2 \mathrm{~nm}$ Fe I line. We show marginalized posterior probability distributions. The results shown in Fig. 8 indicate that the information encoded in the observed data is enough to constrain the characteristics of the magnetic field vector. Except for the case of the azimuth of the field, the marginalized one-dimensional probability distribution functions present an asymmetric non-Gaussian shape, with extended wings. The parameters are constrained by the observations and the inferred values are given in each plot, together with the $68 \%$ confidence interval. We show the two-dimensional distributions as contour plots, where the 68 and $95 \%$ confidence levels are indicated.

The Fe I line at $630.1 \mathrm{~nm}$ results are less reliable. The posterior distributions are much broader than for the $630.2 \mathrm{~nm}$ line and they present strong degeneracies. The elongated shape of the $p\left(B, \theta_{B}\right)$ posterior indicates a certain degree of degeneracy between both parameters. The reason for this behavior is that the $630.1 \mathrm{~nm}$ line is still in the transition from the Zeeman weakfield regime to a saturation regime. As a consequence, the $B$ $\theta_{B}$ degeneracy that we have discussed in Sect. 4.1 introduces problems in the unique determination of the field strength and inclination. These results have been obtained assuming $\sigma=$ $1.5 \times 10^{-3}$ in units of the continuum intensity. This is a relatively large value which allows a relaxed tolerance in the quality of the fit, resulting in increased tolerance in the inferred parameters. Clearly, due to the different magnetic sensitivity, noise differently affects both spectral lines. Since the Zeeman splitting in the $630.2 \mathrm{~nm}$ line is clearly visible, the information about the magnetic field strength is readily available from the peak separation in the Stokes $V$ profile. This separation is much less affected by noise. Once the field strength is fixed, the inclination and azimuth of the field are easily obtained. Contrarily, since the $630.1 \mathrm{~nm}$ line is partially in the weak-field regime, the magnetic field strength has to be obtained from the amplitude of the Stokes
$V$ profile, together with the rest of Stokes parameters. The estimated value of the field strength crucially depends on the value of the tolerance $\sigma$. As a proof of this, we have verified that the shape of the $p\left(B, \theta_{B}\right)$ surface shown in Fig. 10 approximately follows $\cos \theta_{B} \propto 1 / B$ and that the width is related to the tolerance $\sigma$.

The results of both inversions should also be regarded in conjunction, as shown in Fig. 11. If the line formation region of both lines would have been exactly the same, one would expect to find equivalent results from both lines. Since this is not the case (e.g., Shchukina \& Trujillo Bueno 2001), some differences might exist. In spite of this, the posterior distributions clearly overlap in a region of the space of parameters that describes the magnetic field vector. The results clearly demonstrate that the combination of the two lines produces a slight improvement in the restriction of the parameters. However, the result is very similar to what we find using only the line at $630.2 \mathrm{~nm}$. The field azimuth is compatible with a value of $217.4_{-7.8}^{+7.0}$ degrees. We have restricted the range of variation of the azimuth arbitrarily to $[\pi, 2 \pi]$, thus avoiding ambiguities. However, we have verified that the code is able to correctly capture the intrinsic azimuth ambiguity when the range of variation is set to $[0,2 \pi]$. The field inclination given by both lines is consistent with $45.4_{-3.2}^{+3.0}$ degrees, while the magnetic field strength is consistent with $1768.6_{-133.8}^{+165.9} \mathrm{G}$. Figure 11 shows what we consider one of the most appealing properties of the Bayesian method for the inversion of Stokes profiles. It is possible to assess the amount of information given by one spectral line individually and combine many lines in order to investigate whether the added information helps in better constraining the model parameters.

Our results indicate that the information obtained from the $630.1 \mathrm{~nm}$ line alone is low and that it cannot restrict the magnetic field vector for the noise level that we obtain in the observations. We have inverted both lines simultaneously following the same scheme as presented above. We do not show a graphical representation of the results because they are very similar to those inferred from the $630.2 \mathrm{~nm}$ line which can be found in Fig. 8, as also suggested by Fig. 11. We must accumulate information from many spectral lines, with the hope that the combined effect allows us to better constrain the physical parameters (Semel 1981; Socas-Navarro 2004a; Asensio Ramos et al. 2007b).

\section{Concluding remarks}

The framework that we have presented here is very general and is applicable to any existing Stokes inversion code. Once a model that can be used to calculate the emergent Stokes profiles is available, the MCMC method can be used to efficiently explore the posterior probability distribution function. Presently, we are obtaining enormous amounts of Stokes profiles observations from existing ground-based instrumentation like THEMIS (López Ariste et al. 2000), TIP (Martínez Pillet et al. 1999) and POLIS (Beck et al. 2005) and with the space-based instrumentation like the recent mission HINODE. The pressure will be even greater once the new generation of larger solar telescopes like GREGOR and ATST becomes available. Therefore, much effort has been put into developing fast inversion codes that can cope with such large amount of observations. Inversion codes based on PCA (Rees et al. 2000) and artificial neural networks (SocasNavarro 2005) are good candidates for such work.

Our approach here has a different philosophy. We understand that Bayesian inversions cannot compete in speed with these fast algorithms (they cannot even compete with standard inversion 

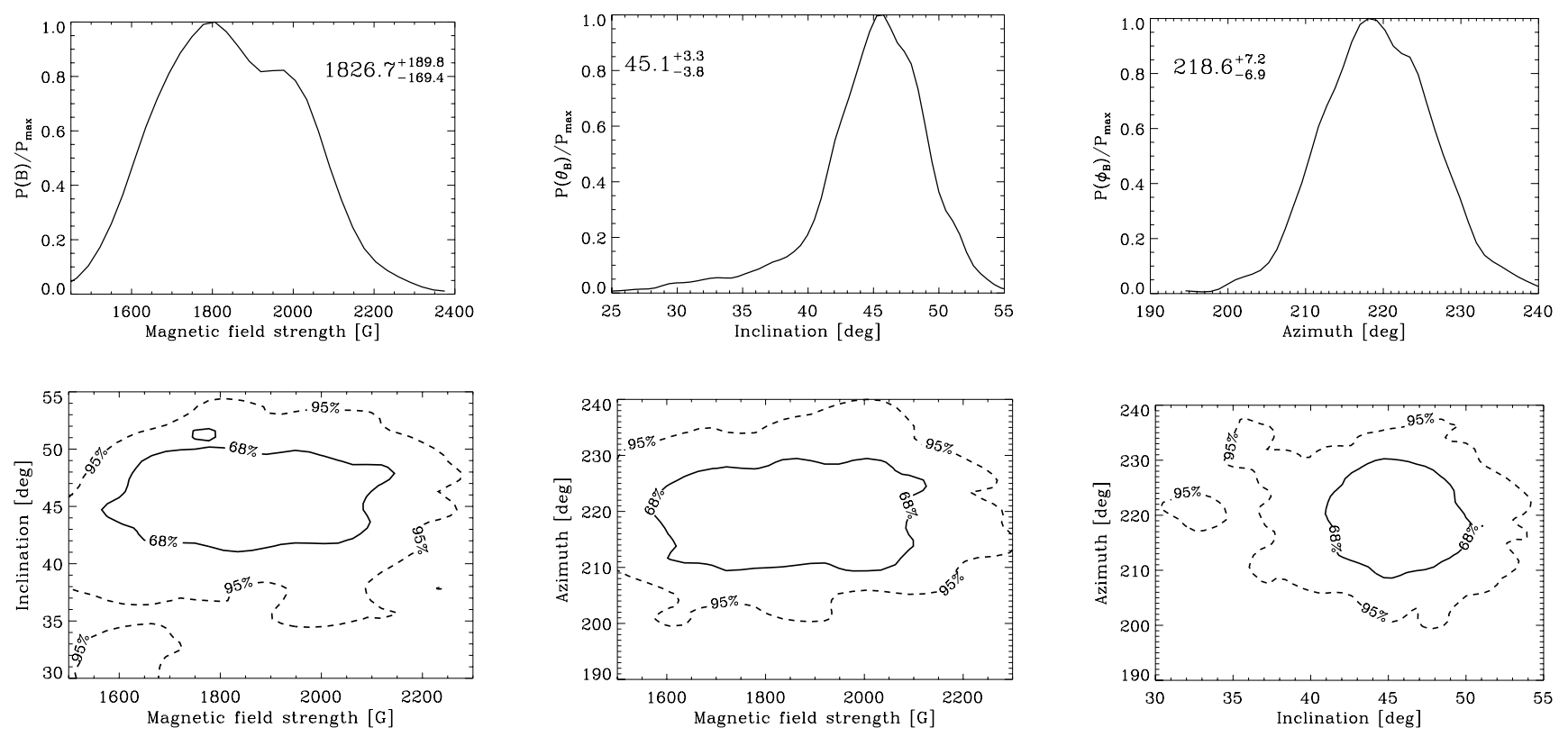

Fig. 8. One-dimensional and two-dimensional marginalized posterior distributions for the parameters that define the magnetic field vector for the inversion of the Fe I line at $630.2 \mathrm{~nm}$. The distributions clearly indicate the presence of a peak belonging to the value of the parameters that produce the best fit. We have indicated in the one-dimensional posteriors the most probable value of each parameter (large arrow), together with the 68\% confidence interval (small arrows). Note the presence of asymmetric confidence intervals. The two-dimensional posteriors have been represented with contours indicating the confidence levels at $68 \%$ (solid line) and $95 \%$ (dashed line).
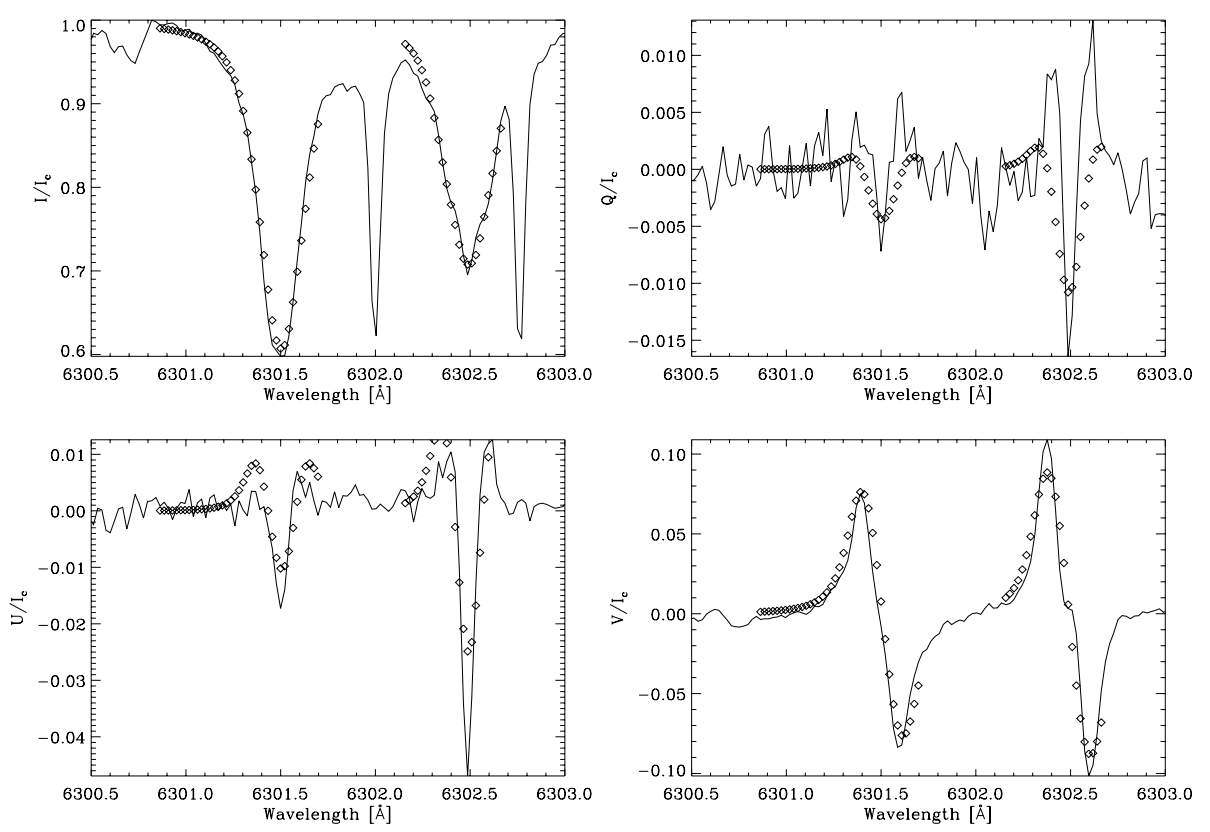

Fig. 9. Stokes profiles observed in the umbra of a sunspot (solid lines). The well known $630.2 \mathrm{~nm}$ Fe I line presents a larger magnetic sensitivity that translates into an enhanced Zeeman splitting. The noise in the observations is of the order of $1.5 \times 10^{-3}$ in units of the continuum intensity. The symbols show the fit obtained when taking the most probable value of the parameters inferred from the Markov chain.

codes based on Levenberg-Marquardt optimization). However, the Bayesian approach is the only one that can be used to investigate in detail the accuracy of inversions, the sensitivity of the parameters to the noise and give confidence intervals to all the inferred parameters. Furthermore, it can be used to rule out a given model due to its lack of ability to fit a given observed Stokes profile. Our approach can make use of the well-developed machinery behind the Bayesian formalism (e.g., Marshall et al. 2006; Liddle 2007). For instance, model selection techniques based on the calculation of the evidence can be introduced. Similarly to the results presented by Asensio Ramos (2006), the simplest model that fits the observations well is preferred to more complicated models (even if they produce a slightly better fit).

In spite of the intrinsic high computational load of the MCMC method, one of its advantages is that it is easily parallelizable. Many Markov chains can be run simultaneously in different isolated threads with no communication between them. Once the chains are finished, they can be combined into a large chain. Since each Markov chain (after the burn-in period) samples from the posterior distribution, we obtain a very large chain that also samples from the posterior distribution. Except for the presence of a burn-in period in each chain, the gain in 

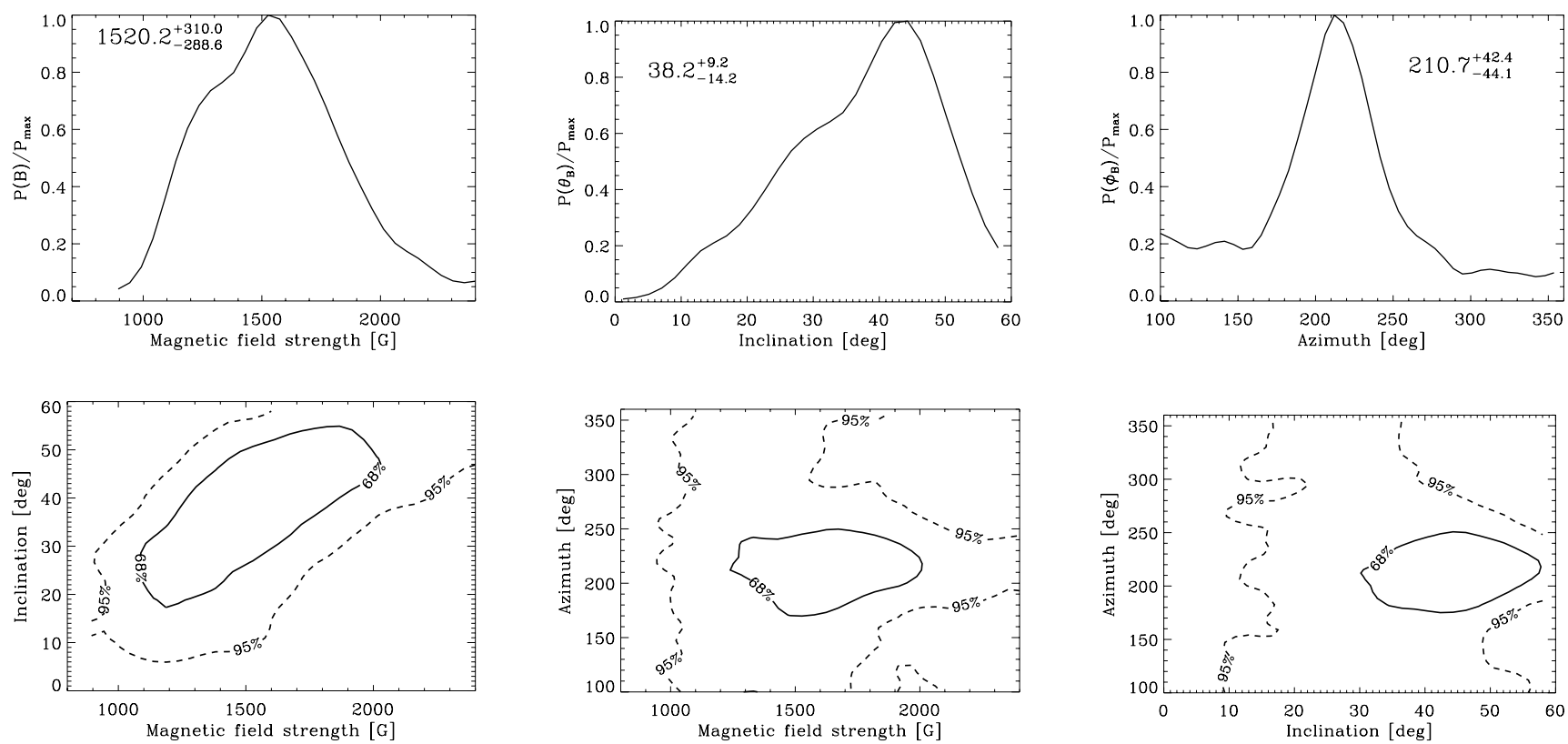

Fig. 10. Same as Fig. 8 but for the Fe I line at $630.1 \mathrm{~nm}$. The two-dimensional representations of the posterior distribution clearly indicate the presence of a degeneracy between the inclination and the magnetic field strength. This translates into very broad one-dimensional marginalized distributions.
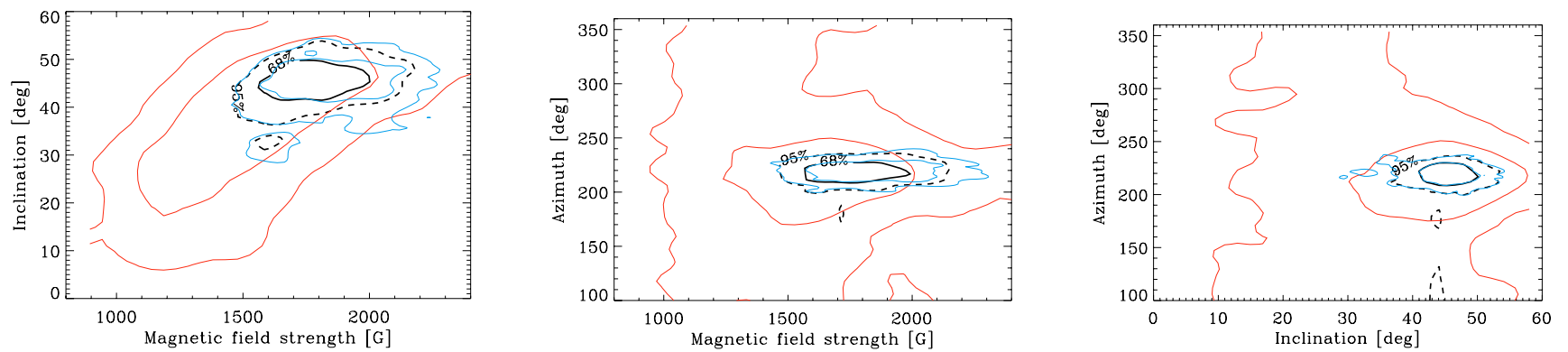

Fig. 11. Combined posterior probability distribution for the 630.1 and $630.2 \mathrm{~nm}$ lines. Note that there is a region of compatibility between both lines, that gives a magnetic field of $\sim 1700 \mathrm{G}$, an inclination of $\sim 45^{\circ}$ and an azimuth of $\sim 220^{\circ}$.

computational time is roughly proportional to the number of threads. A more refined way of parallelization is to start a chain and, after the burn-in period, subdivide it into different threads. At the end, all the threads are combined and we end up with a long chain. In this case, the gain in computational time is slightly larger than in the previous case.

The inversion code for the Milne-Eddington case (Bayes$\mathrm{ME}$ ) is freely available from the authors. The present version of the code is extremely versatile and it has a very good convergence rate. However, we plan to introduce different refinements in the future. The most straightforward is the modification of the proposal density so that non-diagonal elements of the covariance matrix can be taken into account. Although the convergence rate assuming a diagonal covariance matrix is acceptable, this refinement can lead to a reduction in the length of the chains because more structure of the posterior distribution is captured in the proposal density.

Acknowledgements. We thank R. Manso Sainz and A. López Ariste for illuminating discussions. We also acknowledge the help of A. Sainz Dalda for providing umbra profiles. This research has been partly funded by the Ministerio de Educación y Ciencia through project AYA2004-05792.

\section{References}

Asensio Ramos, A. 2006, ApJ, 646, 1445

Asensio Ramos, A., Martínez González, M. J., López Ariste, A., Trujillo Bueno, J., \& Collados, M. 2007a, ApJ, 659, 829

Asensio Ramos, A., Socas-Navarro, H., López Ariste, A., \& Martínez González, M. J. 2007b, ApJ, 660, 1690

Auer, L. H., House, L. L., \& Heasley, J. N. 1977, Sol. Phys., 55, 47

Beck, C., Schmidt, W., Kentischer, T., \& Elmore, D. 2005, A\&A, 437, 1159

Bellot Rubio, L. R. 2006, in Solar Polarization Workshop 4, ed. R. Casini \& B. W. Lites, ASP Conf. Ser., 358, 107

Bommier, V., Landi Degl'Innocenti, E., Landolfi, M., \& Molodij, G. 2007, A\&A, 464,323

Brewer, B. J. \& Lewis, G. F. 2006, ApJ, 637, 608

Brewer, B. J., Bedding, T. R., Kjeldsen, H., \& Stello, D. 2007, ApJ, 654, 551

Casini, R. \& Judge, P. G. 1999, ApJ, 522, 524

Casini, R., Bevilacqua, R., \& López Ariste, A. 2005, ApJ, 622, 1265

Cornish, N. J. \& Crowder, J. 2005, Phys. Rev. D, 72, 043005

Domínguez Cerdeña, I., Sánchez Almeida, J., \& Kneer, F. 2003, A\&A, 407, 741

Dunkley, J., Bucher, M., Ferreira, P. G., Moodley, K., \& Skordis, C. 2005 , MNRAS, 356, 925

Gelman, A., Roberts, G. O., \& Gilks, W. R. 1996, in Bayesian Statistics 5, ed.

J. M. Bernardo, J. Berger, A. Dawid, \& A. Smith, 599

Gelman, A. \& Rubin, D. B. 1992, Statist. Sci., 7, 457

Grossmann-Doerth, U., Keller, C. U., \& Schuessler, M. 1996, A\&A, 315, 610

House, L. L. 1977, ApJ, 214, 632

Illing, R. M. E., Landman, D. A., \& Mickey, D. L. 1975, A\&A, 41, 183

Kashyap, V. \& Drake, J. J. 1998, ApJ, 503, 450

Keller, C. U., Steiner, O., Stenflo, J. O., \& Solanki, S. K. 1990, A\&A, 233, 583 
Khomenko, E. V., Collados, M., Solanki, S. K., Lagg, A., \& Trujillo Bueno, J. 2003, A\&A, 408, 1115

Landi Degl'Innocenti, E. 1992, in Solar Observations: Techniques and Interpretation, ed. F. Sánchez, M. Collados, \& M. Vázquez (Cambridge: Cambridge University Press), 73

Landi Degl'Innocenti, E. \& Landolfi, M. 2004, Polarization in Spectral Lines (Kluwer Academic Publishers)

Lewis, A. \& Bridle, S. 2002, Phys. Rev. D, 66, 103511

Liddle, A. R. 2007, MNRAS, 377, L74

Lin, H. 1995, ApJ, 446, 421

Lites, B. W., Thomas, J. H., Bogdan, T. J., \& Cally, P. S. 1998, ApJ, 497, 464

López Ariste, A. \& Casini, R. 2002, ApJ, 575, 529

López Ariste, A., Rayrole, J., \& Semel, M. 2000, A\&AS, 142, 137

MacKay, D. J. C. 2003, Information Theory, Inference, and Learning Algorithms (Cambridge University Press)

Marshall, P., Rajguru, N., \& Slosar, A. 2006, Phys. Rev. D, 73, 067302

Martínez González, M. J., Collados, M., \& Ruiz Cobo, B. 2006, A\&A, 456, 1159

Martínez Pillet, V., Collados, M., Bellot Rubio, L. R., et al. 1999, in Astronomische Gesselschaft Meeting Abstracts, 15

Mathew, S. K., Lagg, A., Solanki, S. K., et al. 2003, A\&A, 410, 695

Metropolis, N., Rosenbluth, A. W., Rosenbluth, M. N., Teller, A. H., \& Teller, E. 1953, J. Chem. Phys., 21, 1087

Neal, R. M. 1993, Probabilistic Inference Using Markov Chain Monte Carlo Methods (Dept. of Statistics, University of Toronto: Technical Report No. 0506)

Press, W. H., Teukolsky, S. A., Vetterling, W. T., \& Flannery, B. P. 1986, Numerical Recipes (Cambridge: Cambridge University Press)
Rebolo, R., Battye, R. A., Carreira, P., et al. 2004, MNRAS, 353, 747

Rees, D. E., López Ariste, A., Thatcher, J., \& Semel, M. 2000, A\&A, 355, 759 Rubiño-Martin, J. A., Rebolo, R., Carreira, P., et al. 2003, MNRAS, 341, 1084 Ruiz Cobo, B. \& del Toro Iniesta, J. C. 1992, ApJ, 398, 375

Sainz Dalda, A. \& López Ariste, A. 2007, A\&A, submitted

Sanchez Almeida, J. 1997, ApJ, 491, 993

Sanchez Almeida, J., Collados, M., \& del Toro Iniesta, J. C. 1989, A\&A, 222, 311

Sánchez Almeida, J., Domínguez Cerdeña, I., \& Kneer, F. 2003, ApJ, 597, L177 Semel, M. 1981, A\&A, 97, 75

Shchukina, N. \& Trujillo Bueno, J. 2001, ApJ, 550, 970

Sigwarth, M., Balasubramaniam, K. S., Knölker, M., \& Schmidt, W. 1999, A\&A, 349,941

Skumanich, A. \& López Ariste, A. 2002, ApJ, 570, 379

Socas-Navarro, H. 2004a, ApJ, 613, 610

Socas-Navarro, H. 2004b, ApJ, 614, 457

Socas-Navarro, H. 2005, ApJ, 621, 545

Socas-Navarro, H. \& Sánchez Almeida, J. 2002, ApJ, 565, 1323

Stenflo, J. O. 1994, Solar Magnetic Fields. Polarized Radiation Diagnostics (Dordrecht: Kluwer Academic Publishers)

Trujillo Bueno, J. 1999, in Solar Polarization, ed. K. N. Nagendra \& J. O. Stenflo, (Kluwer Academic Publishers), 73

Trujillo Bueno, J. 2001, in Advanced Solar Polarimetry - Theory, Observation, and Instrumentation, ed. M. Sigwarth, ASP Conf. Ser., 236, 161

Westendorp Plaza, C., del Toro Iniesta, J. C., Ruiz Cobo, B., et al. 1997, Nature, 389,47 
A. Asensio Ramos et al.: Bayesian inversion of Stokes profiles, Online Material $p 1$

\section{Online Material}




\section{Appendix A: Markov chain Monte Carlo}

\section{A. 1 Metropolis algorithm}

This approach directly samples the posterior distribution using a Markov chain. The elements of the chain are the vector of parameters $\boldsymbol{\theta}$ that are used to describe each model. The Markov chain is a stochastic process $\left\{\boldsymbol{\theta}_{0}, \boldsymbol{\theta}_{1}, \ldots, \boldsymbol{\theta}_{n}\right\}$ in which each element $\boldsymbol{\theta}_{i}$ only depends on the previous one $\boldsymbol{\theta}_{i-1}$. The key idea of the MCMC method is to choose the next point in the chain depending on the previous point such that the distribution of the chain asymptotically tends to be equal to the posterior distribution, i.e.:

$\lim _{n \rightarrow \infty} p\left(\left\{\boldsymbol{\theta}_{0}, \boldsymbol{\theta}_{1}, \ldots, \boldsymbol{\theta}_{n}\right\}\right)=p(\boldsymbol{\theta} \mid D)$.

Several methods are available, although we will focus on the Metropolis algorithm (Metropolis et al. 1953; Neal 1993) that, in spite of its simplicity, gives extremely good results. The algorithm can be defined as follows:

1. Choose a starting vector of parameters $\boldsymbol{\theta}_{0}$. If some information is available about the value of some of the parameters, it is advantageous to start close to the solution. However, this condition is not mandatory for the convergence of the Markov chain.

2. Calculate the posterior probability given the data $p\left(\boldsymbol{\theta}_{0} \mid D\right)$. This includes the calculation of the priors and the likelihood (including the calculation of the forward modeling problem).

3. Obtain a new vector of parameters $\boldsymbol{\theta}_{i}$ sampling from a proposal density distribution $q\left(\boldsymbol{\theta}_{i} \mid \boldsymbol{\theta}_{i-1}\right)$. We will explain this step more in detail afterwards.

4. Evaluate the posterior probability $p\left(\boldsymbol{\theta}_{i} \mid D\right)$.

5. Evaluate the ratio

$$
r=\frac{p\left(\boldsymbol{\theta}_{i} \mid D\right) q\left(\boldsymbol{\theta}_{i} \mid \boldsymbol{\theta}_{i-1}\right)}{p\left(\boldsymbol{\theta}_{i-1} \mid D\right) q\left(\boldsymbol{\theta}_{i-1} \mid \boldsymbol{\theta}_{i}\right)} .
$$

Admit $\boldsymbol{\theta}_{i}$ in the Markov chain with probability

$$
\beta=\min [1, r] \text {. }
$$

If a point is rejected, include $\boldsymbol{\theta}_{i-1}$ in the chain. 6. Go back to step to 3 .

It has been shown that the previous numerical scheme leads to a Markov chain whose probability distribution converges towards the posterior distribution (e.g., Metropolis et al. 1953). The advantage with respect to the brute force approach is that the number of evaluations of the posterior distribution no longer increases exponentially with the number of parameters, but linearly. As a consequence, we can treat much more complicated problems with a reduced computational effort. The reason for this behavior is that since the chain is sampling the underlying posterior distribution, the regions of larger probability are evaluated more times. The proposal density distribution is usually chosen to be symmetric, thus $q\left(\boldsymbol{\theta}_{i} \mid \boldsymbol{\theta}_{i-1}\right)=q\left(\boldsymbol{\theta}_{i-1} \mid \boldsymbol{\theta}_{i}\right)$. As a consequence, the ratio to be evaluated in step 5 simplifies to $r=p\left(\boldsymbol{\theta}_{i} \mid D\right) / p\left(\boldsymbol{\theta}_{i-1} \mid D\right)$.

\section{A.2 The proposal density}

The key ingredient of the Metropolis MCMC algorithm is the proposal density. In the ideal case, one should choose $q\left(\boldsymbol{\theta}_{i} \mid \boldsymbol{\theta}_{i-1}\right)$ as close to the posterior distribution as possible. In the limiting case that the proposal distribution exactly matches the posterior one, one is carrying out a perfect sampling: more samples are performed in the regions of larger probability. Consequently, all the proposed steps will be included in the Markov chain. This case is obviously unrealistic because it assumes that our aim (i.e., the evaluation of the posterior distribution) already has been achieved.

The power of the MCMC scheme lies in the fact that even naïvely chosen proposal densities lead to an algorithm that efficiently samples from the posterior distribution. However, it is also true that a careful selection of the proposal density greatly improves the convergence rate of the algorithm. Common proposal densities include Gaussian or uniform distributions centered on the current value of the parameters to propose a new value of the parameters. In our case, we have chosen a combination of Gaussian and uniform distributions. Both cases lead to a symmetric proposal density. For the initial $N_{\text {unif }}$ steps of the chain, we propose parameters following a uniform distribution in each parameter. The limits of the uniform distribution are free parameters chosen to be equal to their range of variation. The minimum values for all the parameters are put in the vector $\boldsymbol{\theta}^{\mathrm{min}}$ while the maximum values are included in the vector $\boldsymbol{\theta}^{\max }$. Then:

$q\left(\boldsymbol{\theta}_{i} \mid \boldsymbol{\theta}_{i-1}\right) \sim U\left(\boldsymbol{\theta}^{\min }, \boldsymbol{\theta}^{\max }\right)$

After the first $N_{\text {unif }}$ steps, some information about the posterior probability is known. Therefore, statistical properties like the covariance matrix $\mathbf{C}$ can be estimated. At this point, we change to a Gaussian proposal density centered on the current value of the parameters. Ideally, one should propose with the following distribution:

$q\left(\boldsymbol{\theta}_{i} \mid \boldsymbol{\theta}_{i-1}\right) \sim \exp \left[-\frac{\alpha}{2} \boldsymbol{u}^{\dagger} \mathbf{C}^{-1} \boldsymbol{u}\right]$

where $\boldsymbol{u}=\boldsymbol{\theta}_{i}-\boldsymbol{\theta}_{i-1}, \boldsymbol{u}^{\dagger}$ stands for the transpose of the $\boldsymbol{u}$ vector and $\alpha$ is a constant whose meaning will be discussed later. Sampling from such a proposal density would require the diagonalization of the covariance matrix due to the matrix inversion (e.g., Dunkley et al. 2005). This proposal density is very useful for problems in which strong degeneracies are present in the problem, so that the posterior distribution shows very elongated maxima. However, in the first version of our inversion code, we neglect the non-diagonal elements of the covariance matrix. We have verified that this approximation gives extremely good results in our case (in spite of the degeneracies present in the problem). The inclusion of non-diagonal terms in the covariance matrix is left for future revisions of the code.

When we only take into account the diagonal elements of the covariance matrix, the proposal of each parameter can be done independently of the rest of parameters. Random numbers following a normal distribution with unit variance are picked and the proposed value for each parameter is obtained by multiplying them by their corresponding variances. The variances are updated after a fixed number of iterations of the Markov chain. It is not necessary to use the whole Markov chain to estimate the variances, because the following updating rule can be applied to update the variance of parameter $i$ at step $n$ :

$$
\begin{aligned}
\sigma_{i}^{2}(n)= & \frac{n-1}{n} \sigma_{i}^{2}(n-1) \\
& +\frac{\left[\theta_{i}(n)-\overline{\theta_{i}}(n-1)\right]^{2}}{(n+1)^{2}}+\frac{\left[\theta_{i}(n)-\overline{\theta_{i}}(n)\right]^{2}}{n},
\end{aligned}
$$

where $\theta_{i}(n)$ is the value of the proposed parameter, $\bar{\theta}_{i}(n-1)$ is the average of the parameter $i$ taking into account the first $n-1$ elements of the chain, while $\overline{\theta_{i}}(n)$ takes also into account element 

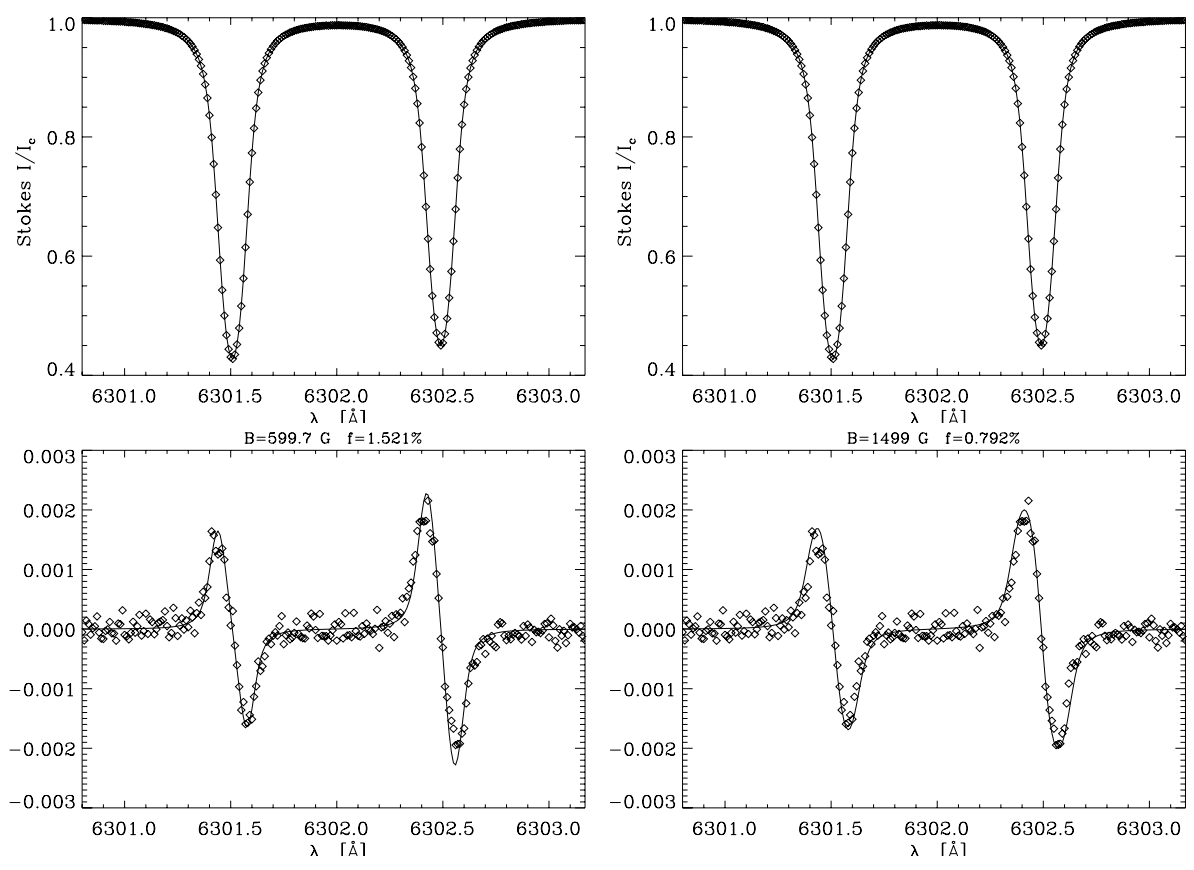

Fig. B.1. Synthetic profiles with added noise (diamonds) together with two different synthetic profiles corresponding to models presenting magnetic field strengths differing by $900 \mathrm{G}$ (solid lines). Since both models fit the profiles below $1 \sigma$, there is no objective reason to favor one of them. $n$ in calculating the average. The average can also be updated following the rule:

$\overline{\theta_{i}}(n)=\overline{\theta_{i}}(n-1)+\frac{\theta_{i}(n)-\overline{\theta_{i}}(n-1)}{n+1}$.

The constant $\alpha$ is used to tune the convergence process. It has been demonstrated (Gelman et al. 1996; Dunkley et al. 2005) that, in order to efficiently sample from a posterior distribution, the acceptance rate of models should be of the order of $25 \%$. We use $\alpha$ to shrink or broaden the proposal density so that such an acceptance rate is assured. We have verified with an extensive test phase that this technique behaves consistently and the chain rapidly samples the posterior distribution.

\section{A.3 Convergence}

The convergence of the Markov chain is a critical issue (e.g., Gelman \& Rubin 1992; Lewis \& Bridle 2002). A chain is said to be converged when the statistical properties of its elements reflect with "enough accuracy" the statistical properties of the underlying distribution that is being sampled. A problem arises for what "enough accuracy" means. Great efforts have been put into the development of powerful convergence tests (e.g., Gelman \& Rubin 1992). The key ingredient in dictating the convergence rate is the proposal density distribution. One of the most widely applied methods of convergence testing is the one proposed by Gelman \& Rubin (1992). The main drawback is that it works by generating several Markov chains with random initial points. A posterior analysis of their statistical properties helps us to distinguish when a chain is sampling from the posterior distribution. At this point, the elements of the chain can be used to obtain information about the statistical properties of the posterior distribution that we are sampling. Our code uses the alternative of Dunkley et al. (2005) to test for convergence. It is based on the idea that the Fourier power spectrum of a the Markov chain would be flat and equal to the variance of the underlying distribution if complete convergence is obtained. However, the chain can be considered as converged under much less restrictive conditions (see Dunkley et al. 2005, for details).

At the beginning of the MCMC algorithm, the chain typically proposes large jumps through the parameter space until the regions of high posterior probability distribution are located. This is especially true when the initial point of the chain is very far away from the regions of large posterior density. The chain, once it has migrated to these regions, proposes smaller jumps. The initial steps of the chain are not representative of the underlying posterior $p(\theta \mid D)$. They are usually known as the "burnin" of the chain and these elements are typically discarded. Following Dunkley et al. (2005), one easy way to locate the number of elements of the "burn-in" is to locate the maximum value of the posterior $p_{\max }$ and discard the first elements of the chain until $p(\theta \mid D) / p_{\max }>f$, with $f \sim 0.1-0.2$. When the initial point of the chain is close to the high probability region, this scheme leads to a "burn-in" of a few (or even zero) elements.

\section{Appendix B: Profiles}

According to Fig. 4, fields above $500 \mathrm{G}$ and below $1800 \mathrm{G}$ fit the synthetic profile with added noise with a precision smaller than $1 \sigma$. When this constraint is relaxed to $2 \sigma$, the fields can be even larger or smaller. Using the three plots of the upper panel of Fig. 4, it is possible to detect a large number of combinations where fits inside the $68 \%$ confidence level can be obtained with sub-kG and $\mathrm{kG}$ fields. As an example, we show in Fig. B.1 a fit to the synthetic Stokes profiles with added noise with a field of $600 \mathrm{G}$ and with a field of $1500 \mathrm{G}$. There is no objective reason to prefer one fit over the other within a $1 \sigma$ uncertainty, as is consistent with the results presented in Fig. 4. Note that this result was pointed out for the first time by Martínez González et al. (2006). 